\title{
OSTEOCONDRITIS DISECANTE: UNA REVISIÓN BIBLIOGRÁFICA DE ACTUALIDAD
}

\author{
Alonso Caravaca, Adrián
}

Resumen: La osteocondritis disecante es una enfermedad que aparece más frecuentemente en varones jóvenes, a nivel de la rodilla, y con mejor pronóstico a esas edades tempranas. Su etiopatogenia se considera multifactorial, influyendo la interacción de aspectos constitucionales y del metabolismo, con otros mecánicos (siendo éstos últimos, como los microtraumatismos, de mayor relevancia actualmente). El dolor a la carga es el síntoma más frecuente (80\%), y para su diagnóstico nos apoyaremos en radiografías simples, Tomografía Computarizada (TC) y Resonancia Magnética (RM), pero el gold-standard es artroscópico. En cuanto al tratamiento de la enfermedad, primero se intenta actuar con terapias conservadoras, y posteriormente cobra importancia la cirugía. Existen dos bloques de tratamiento quirúrigo: el reparativo (técnicas de fijación in situ, micro-/nanoperforaciones) y el sustitutivo (injerto óseo autólogo, injertos osteocondrales de diversa naturaleza, cultivos de condrocitos y, como última opción la artroplastia). En la literatura existe gran discusión acerca del manejo, que depende en su mayoría de factores como el tamaño de la lesión, la demanda funcional del paciente o la edad, sin embargo, no hay consenso, y cada autor suele proponer un algoritmo diferente. Tampoco se ha encontrado en la literatura evidencia lo suficientemente potente que permita recomendar un tratamiento en concreta, siendo cierto que el cultivo autólogo de condrocitos parece una opción muy prometedora cuando se desarrolle por completo. Si hay algo seguro en esta enfermedad, es que hay que tratar primero la deformidad que la ocasiona (en caso de haberla), y adecuar e individualizar los tratamientos, de manera que no sobre-/infratratemos a los pacientes.

Palabras clave: ostecondritis disecante, osteocondritis.

${ }^{1}$ Corresponding Author: Alonso-Caravaca, A, La

Fe Hospital.

adrian.alonso.caravaca@gmail.com

Av Fernando Abril Martorell 106, Valencia,

España. CP: 46026

${ }^{2}$ La Fe Hospital. Av Fernando Abril Martorell

106, Valencia, España. CP: 46026 


\section{INTRODUCCIÓN}

Sabemos que las superficies diarticulares presentan cartílago hialino, y que éste se compone de condrocitos (células que viven en condiciones límite, como un bajo contenido en oxígeno) y la matriz, con proporciones variables de colágeno, elastina y otras sustancias. Justo debajo de ese cartílago encontramos el hueso subcondral, con características de hueso trabecular/esponjoso. En la enfermedad conocida como osteocondritis disecante $(O C D)$, se produce una degeneración del hueso subcondral por diferentes mecanismos que terminan por dificultar la nutrición del cartílago articular. Esta disrupción puede generar afectación cartilaginosa, necrosis ósea y finalmente, la liberación del fragmento por fractura del hueso subcondral, es decir, se producen cuerpos libres intraarticulares. La consecuencia final será la artrosis, que en la mayoría de los casos aparece precozmente. Las localizaciones más fracuentes son la rodilla, el tobillo y el codo (por orden de incidencia).

\section{HISTORIA}

Antes de abordar la enfermedad, y a modo de curiosidad, haremos un inciso para concoer el origen del término osteocondritis.

La primera descripción de una patología que podría entenderse como OCD, la realizó en 1558 Ambroise Paré, el "Padre de la Cirugía" francés, ${ }^{(1)}$ al escribir sobre la extripación de cuerpos libres dentro de las articulaciones. Sin embargo el término OCD fue acuñado por Franz König en 1887, en su artículo "The Classic On Loose Bodies in the Joint". En ella, elaboraba un monólogo acerca de los cuerpos intraarticulares libres en el codo y de rodilla. Describía pacientes con una afectación articular de características inflamatorias, cuya sintomatología cedía una vez de extraían los cuerpos libres, que encontraba de forma casual en algunas de sus cirugías ${ }^{(2)}$.

\section{ETIOPATOGENIA}

Existen muchas teorías al respecto, pero ninguna tiene la fuerza suficiente por sí sola, como para considerarse la causa. El siguiente algortimo, propuesto para la rodilla por Andriolo $\mathrm{L}$. et al. explica la necesaria interacción entre todos los factores que iremos desglosando uno por uno, y en el que influyen tanto mecanismo biológicos como mecánicos ${ }^{(3)}$ [Figura 1].

Factores Biológicos. Estos tres factores acaban afectando al remodelado óseo.

- Factores genéticos como la proteína del gen ACAN, o mutaciones del colágeno.

- Factores endocrinos en relación con cambios en la hGH o al metabolismo de la vitamina D. Estas alteraciones generalmente se encuadran dentro de síndromes sistémicos.

- Teoría de déficit de osificación endocondral: este tipo de osificación es el que se da en los huesos largos. En la OCD se produciría una conexión incompleta entre los sistemas vasculares del centro de osificación secundario, que podría dar lugar a un 
hueso trabecular más débil, incapaz de resistir fuerzas pequeñas. Hay que tener cuidado, porque en caso de pacientes jóvenes con las fisis activas, se puede confundir con variantes de la osificación normal ${ }^{(4)}$.

Factores Mecánicos. Provocan traumatismos en el hueso subcondral.

- Microtrauma repetido, pues se ha visto que un solo macrotrauma no se asocia con esta patología.

- Compresión y choque de la espina tibial durante la rotación interna de la rodilla mientras se produce una carga con flexión de la misma. Esta teoría fue la defendida por Fairbank en 1933.

- Alteraciones biomecánicas causadas iatrogénicamente (meniscectomía) y otras constitucionales (genu recurvatum, aplanamiento de los cóndilos, agenesia de cruzados, desalineaciones en varo/valgo o pendiente tibial aumentada).

- Menisco discoide como particularidad de la rodilla, que produce sobrecarga del hueso subcondral favoreciendo la liberación del fragmento a la articulación.

Estos traumatismos suman en la cascada etiológica, provocando que el hueso subcondral y el cartílago se isquemien y finalmente se fracturen. Para apoyar la teoría de la necrosis subcondral Shea at al. estudian varias muestras histológicas $y$ detectan que ésta pueda ser secundaria a isquemia de los vasos sanguíneos epifisarios terminales ${ }^{(5)}$. Muchas de estas teorías se podrían extrapolar a otras localizaciones.

En el mencionado artículo de Andriolo L., refleja que a lo largo del tiempo, diversos autores han ido describiendo lo que a su parecer y en función de la evidencia, era el origen de esta patología. Sin embargo, en los últimos años, se ha producido un gran repunte que orienta el mismo hacia factores mecánicos que acaban fracturando el hueso subcondral [Figura 2].

Para terminar este apartado, diremos que hay algo que seguro no encontramos en la histología de los cuerpos libres: la inflamación. Barrie en 1984 ya desmintió que (a pesar de la clínica inflamatoria descrita por König), en la histología, no se encontraban hallazgos inflamatorios.

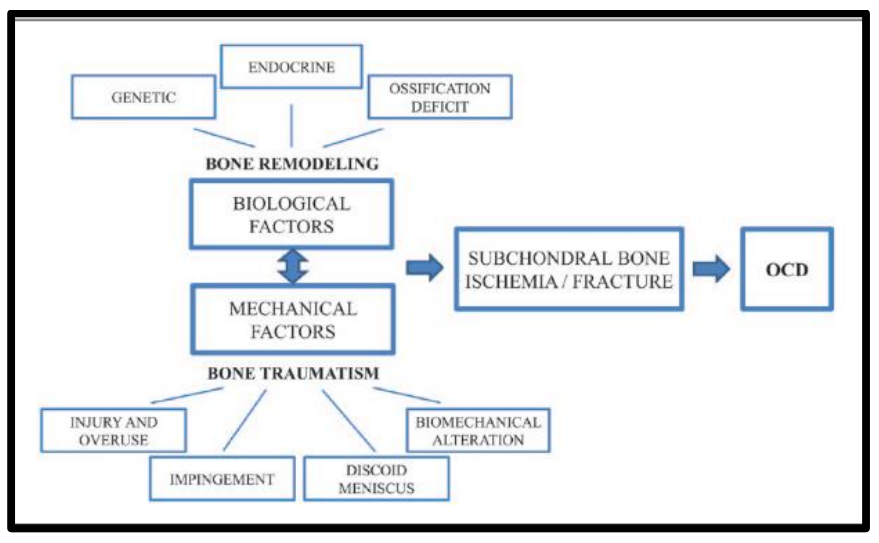

Figura 1. Mecanismo etiopatogénico de la osteocondritis disecante de rodilla, propuesto por Andriolo L., et al. 


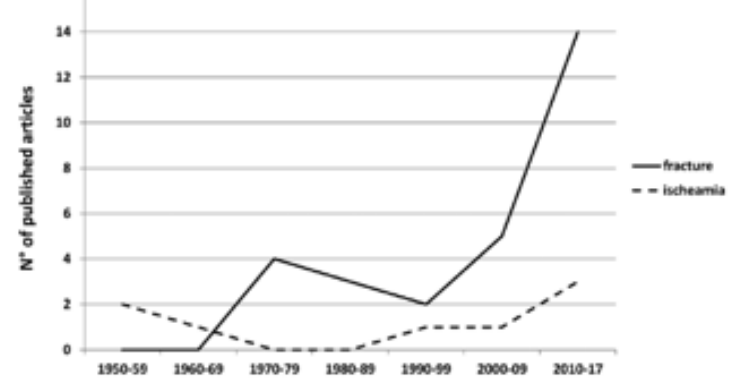

Figura 2. Evolución de las publicaciones científicas sobre la estiología de la osteocondritis.

\section{DIAGNÓSTICO}

Sospechar esta patología va a ser bastante complejo, por ello, se resumen en la Tabla 1 las características epidemiológicas más relevantes que presentan los pacientes, para cada una de las localizaciones de OCD. Destacar que es un patología de hombres adolescentes, en su mayoría, y que la OCD de tobillo es la única que parece no tener predilección por sexo, es más, en algunas series comentan cierta predilección por el sexo femenino. También para el tobillo, sí que se ha encontrado un número de casos no despreciables de OCD como secuela de esguince de tobillo traumático (generalmente esto provoca el desplazamiento de un fragmento ya patológico que hace a la OCD sintomática en el $6.8 \%$ de los casos que generalmente se producen en la cúpula astragalina lateral).

\section{ANAMNESIS Y EXPLORACIÓN FÍSICA}

Lo primero que tenemos que tener en cuenta para sospechar y diagnosticar OCD es hacer una buena anamnesis preguntando por traumatismo reciente, incremento de nivel de actividad, existencia de lesiones previas o la presencia de síntomas mecánicos.

En cuanto a la clínica que presentarán los pacientes, lo más frecuente será dolor a la carga (80\%; de tipo intermitente, mecánico y mal localizado) que puede estar presenta hasta 14 meses antes del diagnóstico. En otros casos pueden cursar con tumefacción (20\%), crepitación, limitación movimiento y bloqueos (cuerpos libres), lo cual habla de que el fragmento será inestable y la enfermedad ya está avanzada, llegando a debutar de esta forma en el $20 \%$ de los casos.

Existen particularidades según la localización:

- Rodilla - test de Wilson: se coloca al paciente en la camilla con la piernas colgando a $90^{\circ}$. Pedimos al paciente que extienda la rodilla mientras sujetamos con el pie para hacer rotación interna. Normalmente el paciente sentirá dolor a unos $30^{\circ}$ (por pinzamiento de la espina), desapareciendo una vez esté en rotación neutra o externa. Se ha visto que este signo es bastante inespecífico (positivo en el $16 \%$ ) y no tiene validez clínica según la evidencia. Habrá que hacer siempre diagnóstico diferencia con patología fémoro-patelar $y$ meniscal.

- Codo - pronosupinación: dolor a la palpación de articulación radiocapitelar durante la prono-supinación con el codo en extensión. En este caso habrá que hacer un diagnóstico diferencial con epicondilitis y fracturas de cabeza de radio. 


\begin{tabular}{|c|c|c|c|}
\hline INCIDENCIA & 6-29 casos/100.000 hab. /año & 0.7-8.9 casos/100.000 hab. /año & 2.2-3.8 casos/100.000 hab. /año \\
\hline EDAD & \multicolumn{3}{|c|}{ 12-19 años } \\
\hline LOCALIZACIÓN & $\begin{array}{l}\text { Cóndilo femoral medial } \\
\text { (porción posterolateral) }\end{array}$ & $\begin{array}{c}\text { Carilla articular medial del } \\
\text { astrágalo }\end{array}$ & Cóndilo humeral (capitellum) \\
\hline LATERALIDAD & \multicolumn{3}{|c|}{ Miembro dominante } \\
\hline SEXO & कా $\gg \gg \&$ & कా $\leq 8$ & कு> \\
\hline RAZA & Negra & Caucásica & Caucásica \\
\hline ASOCIACIÓN & Microtraumatismo repetido & Esguince de tobillo traumático & Microtraumatismo repetido \\
\hline
\end{tabular}

Tabla 1. Epidemiología de la OCD según la localización. (6-8)

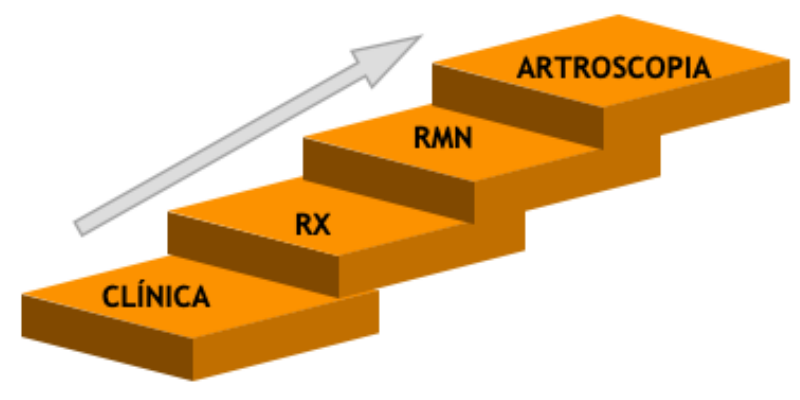

Figura 3: algoritmo diagnóstico sugerido.

\section{RADIOLOGÍA}

Esta herramienta es básica en el quehacer diario del cirujano ortopédico. A continuación se mostrarán imágenes de pacientes diagnosticados de OCD mediante radiografías simples o TC [Figuras 4 y 5 ]. Es importante valorar la articulación contralateral para comparar y percatarse de alteraciones mínimas.

- Rodilla - proyecciones AP, lateral y en túnel/"notch"/Roosemberg: esta última es la mejor para valorar la porción interna y posterior de los cóndilos femorales (se trata de una proyección
AP a $45^{\circ}$ de flexión), así como es utilizada también para la valoración de la artrosis.

- Tobillo - $\mathrm{AP}$, lateral y en mortaja: también en este caso, la proyección específica en mortaja permite valorar al completo la superficie articular.

- Codo- $\mathrm{AP}$, lateral y $\mathrm{AP}$ a $45^{\circ}$ de flexión: solo son positivas en el $66 \%$ de los casos y habrá que hacer un diagnóstico diferencial con la enfermedad de Panner (osteocondrosis idiopática y atraumática el capitellum, generalmente en pacientes menores de 10 años y que es autolimitada.

El TC, siendo una de las herramientas más importantes en el diagnóstico de fracturas óseas, no está sistematizado en el diagnóstico de OCD. Apenas hay artículos actuales que relacionen el uso de TC en la OCD, y es principalmente porque la enfermedad se da en pacientes jóvenes con las fisis abierta y grandes proporciones de cartílago, poco visualizable mediante el TC. Sin embargo, el estudio de van den Ende $^{(11)}$ concluye que el TC es la prueba 
que diagnostica mejor los cuerpos libres intraarticulares en la OCD de codo, y que sería extrapolable también a otras localizaciones.

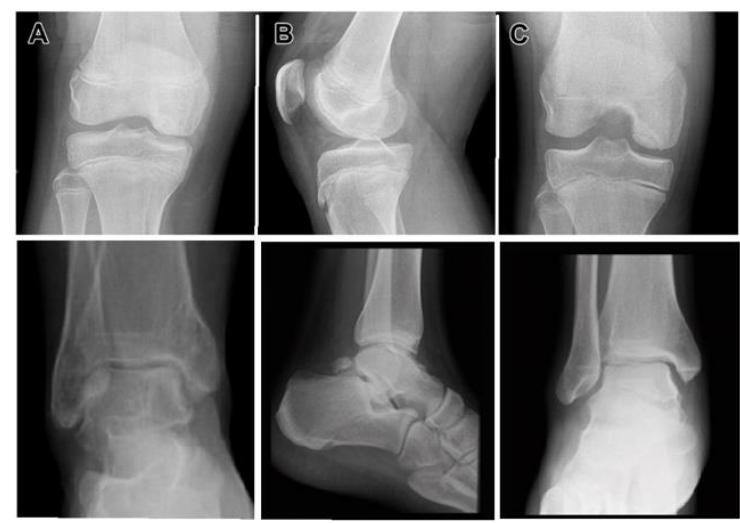

Figura 4. Proyección AP (A), lateral (B) y específica (C; túner arriba, mortaja debajo) en $O C D$ de rodilla y tobillo. Imágenes obtenidas del trabajo de Grimm N., et al. (9)

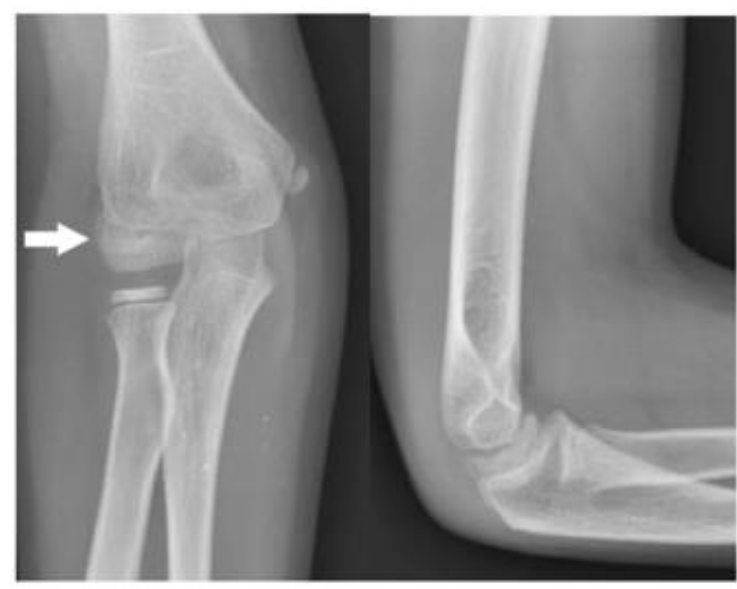

Figura 5. Enfermedad de Panner del codo. Vigilar el diagnóstico diferencial de OCD. Imágenes obtenidas del trabajo de García Cañas R., et al. ${ }^{(10)}$
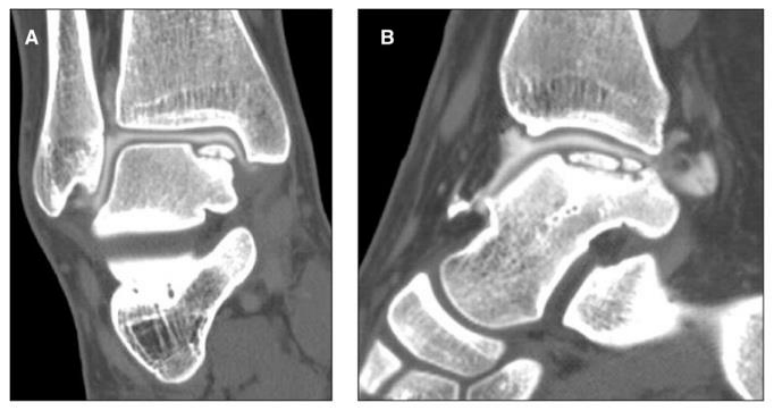

Figura 6. TC de tobillo con contraste intraarticular. Podemos suponer que no hay rotura del cartílago a pesar de la lesión osteocondral que se evidencia ya que el contraste no fuga por debajo de la lesión. Imágenes obtenidas del trabajo de Bauer K.L., \& Polousky, J. D. (12)

\section{RESONANCIA MAGNÉTICA}

Es una de las exploraciones complementarias más útiles para valorar la estabilidad de la lesión, y además se han establecido una serie de criterios para ello(12). En resonancia magnética, mucho más sensible para el diagnóstico de osteocondritis, se usa una secuencia potenciada en T2 (el líquido se ve blanco), de esta forma observaremos el edema circundante a la lesión así como un borde hiperintenso característico (en condiciones normales, en esta secuencia, tanto hueso subcondral como cartílago normales, presentan baja señal). Por lo tanto, una lesión será inestable cuando presente alguna de las siguientes características:

- Quistes subcondrales

- Lesión bien delimitada

- Alta intensidad de señal en T2

- Fractura del cartílago suprayacente

Esta prueba presenta un $100 \%$ de sensibilidad para lesiones en el paciente 
adulto, sin embargo, en pacientes jóvenes (la mayoría de los afectados), ésta se reduce al $11 \%$ para la OCD juvenil de rodilla.
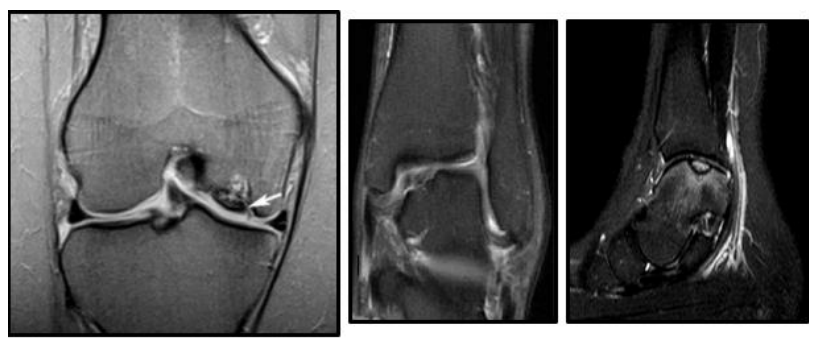

Figura 7. RMN de lesión inestable en visión coronal de rodilla (izquierda) y en visión coronal y sagital de tobillo (centro/derecha). Imágenes tomas de los trabajos de Grimm N., et al. (9) y Bauer K.L., \& Polousky, J. D. (12)

\section{ARTROSCOPIA}

La artroscopia es el método principal para establecer el diagnóstico a día de hoy, sin embargo, no existe una clasificación única para todas las localizaciones descritas, ni tampoco consenso dentro de cada una de ellas. Hablaremos de las más utilizadas o las que más relevancia tienen a día de hoy:

\section{Clasificación de Berndt \& Harty (1959) ${ }^{(13)}$.} Esta clasificación se estableció para la cúpula astragalina en la articualción del tobillo por medio de radiografías simples, y después fue revisada para artroscopia. Consta de cuatro estadíos según la estabilidad de la lesión [Figura 8]:

- Estadío 1. Fractura osteocondral por hundimiento.

- Estadío 2. Fragmento unido mediante puentes óseos.

- Estadío 3. Fragmente libre no desplazado.

- Estadío 4. Fragmento desplazado.

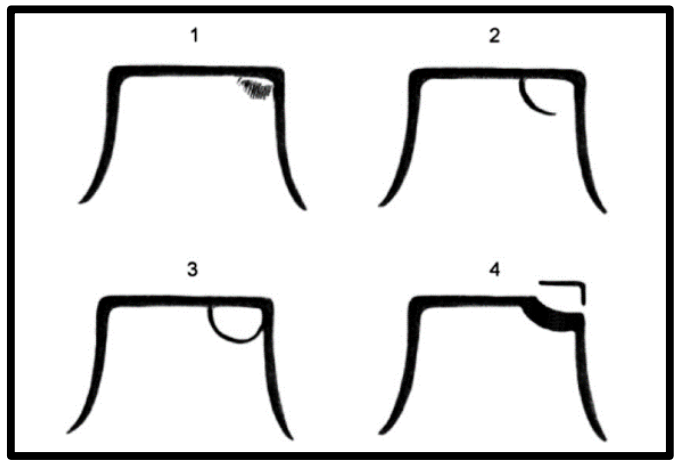

Figura 8. Clasificación de Berndt \& Harty (1959).

Clasificación de la International Cartilage Repair Society (ICRS; 2003) (14). Esta clasificación es la más actualizada hasta el momento y de las más utilizadas a la hora de valorar defectos cartilaginosos, aplicable también en el caso de la OCD, divide en 5 grados en función de la profundidad del defecto [Figura 9]:

- Grado 0. Cartílago normal.

- Grado 1. Afectación superficial. Artroscópicamiente podríamos notar el cartílago intacto pero ablandado.

- Grado 2. Solución de continuidad del cartílago de $<50 \%$ de la profundidad. Tanto el estadío 1 como el 2, son estables cuando se testan en la artrosocpia. Es decir, son lesiones de bajo grado.

- Grado 3. Afectación de más de la mitad del grosor del cartílago. En la artroscopia veríamos una discontinuidad completa pero con fragmento no dislocado.

- Grado 4. Defectos que llegan hasta el hueso subcondral trabecular. Generalmente también encontramos los cuerpos libres durante la artroscopia. 

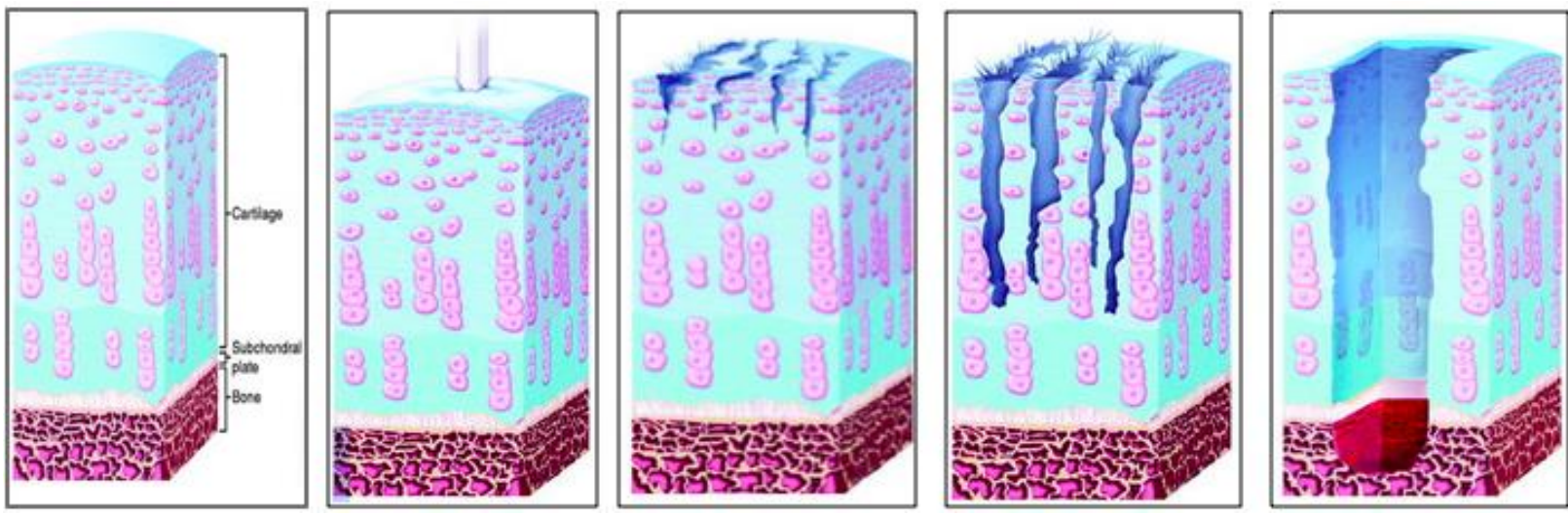

Figura 9. Clasificación ICRS. Grado 0 (arriba), resto (debajo) en orden de gravedad (de izquierda a derecha).

\section{Clasificación del Reseach in Osteochondirtis of the Knee (grupo ROCK; 2016) ${ }^{(15)}$. En vista} de la gran diversidad de clasificaciones que encontramos en la bibliografía (cada una basada en una herramienta diagnóstica), el grupo ROCK ha intentado crear una clasificación artroscópica que pueda servir de base para predecir resultados. Se basa en si la lesión es móvil o no, así como en las características de la misma una vez se realiza la artroscopia. Ha sido validada, y presenta buena concordancia intra e interobservador.

\section{TRATAMIENTO}

Entramos en la sección más controvertida de la revisión. Existen infinidad de técnicas para el tratamiento de lesiones condrales y osteocondrales, por lo que las englobaremos en función de la naturaleza del tratamiento (conservador, reparador o reconstructivo). Si bien estas lesiones se suelen tratar, a veces con un seguimiento $y$ posteriormente, evaluar el desarrollo de la lesión osteocondral, es suficiente. ${ }^{(16)}$

A la hora de elegir un tratamiento u otro nos basaremos principalmente en esta cuatro características: edad del paciente, tamaño de la lesión, actividad del paciente, y estabilidad de la lesión. A grandes rasgos, en aquellos con sintomatología pero sin afectación articular, además en pacientes con inmadurez esquelética (pues los pacientes jóvenes tienen mejor pronóstico) y por supuesto, fragmentos estables, se puede optar por tratamiento conservador. Si este, falla se optará por tratamiento quirúrgico, que en muchos caso, es la principal indicación ${ }^{(17)}$.

\section{TRATAMIENTO CONSERVADOR ${ }^{(18)}$}

Éste será el tratamiento de elección en pacientes jóvenes, con cartílago intacto o lesiones $<2 \mathrm{~cm}^{2}$, y que se ubiquen en la localización clásica para cada articulación, y aunque falta evidencia potente sobre este tema, suelen resolverse a los 6 meses de tratamiento. Dentro de este bloque se incluyen los siguientes tratamientos, sin que ninguno haya probado superioridad sobre otro, soliéndose usar de forma conjunta (tampoco han demostrado eficacia):

- Fisiokinesoterapia. Consiste en calor local y/o terapias manuales. 
- Fortalecimiento muscular.

- Restricción de la actividad física.

- Terapias físicas. Ionoforesis, US, ondas de choque...

- Inmovilicación diminución/limitación completa de la carga. En el caso del codo, no se recomienda la inmovilización para evitar rigideces.

\section{TRATAMIENTO QUIRÚRGICO}

Antes de comenzar con el tratamiento quirúrgico de las lesiones de $O C D$, se debe comentar que no existen criterios establecidos para decantarse por uno u otro, y que será la experiencia del cirujano, y en muchos casos, las características del paciente y la lesión, los que nos harán decantarnos por una opción determinada. En la figura 10 podemos observar la estrategia de tratamiento ante lesiones condrales y oteocondrales propuesto por Vaquero y Forriol (2012) teniendo en cuenta diversas características ${ }^{(19)}$.

Tratamiento reparador. A continuación describiremos tratamientos que buscan reparar el defecto creado por la OCD, sobre todo en pacientes con stock óseo suficiente, y lesiones, en su mayoría estables.

Fijación in situ ${ }^{20)}$. Este tratamiento busca fijar el fragmento osteocondral al hueso del que procede. Se puede realizar por medio de dos vías sin que se haya probado que una sea mejor que la otra:
- Transarticular, por vía artroscópica.

- Retroarticular, con control fluoroscópico. Presentan mayor dificultad técnica.

La fijación in situ con tornillos (canulados o no) del cartílago articular se prefiere para casos de OCD inestable (siempre que haya hueso subyacente suficiente), incluso con agujas de Kirschner. A veces es necesario retirarlos y comprobar la curación con un segundo tiempo.

Microperforaciones (21): Esta opción terapéutica se basa en intentar revitalizar el lecho sobre el que asienta la lesión osteocondral. En primer lugar se desbrida artrocópicamente el cartílago y después se realizan perforaciones de unos $3 \mathrm{~mm}$ de profundidad a lo largo del hueso subcondral de la zona patológica para conseguir un sangrado controlado. Actualmente se está abogando más por las nanoperforaciones, más finas $y$ profundas (hasta $9 \mathrm{~mm}$ ) que consiguen llegar mejor a la médula ósea del hueso largo, que proporcionará células hematopoyéticas que podrían acabar generando una matriz condrosimilar. Además, a esta técnica podemos asociar otras para mejorar los resultados, como muestra la figura 11 , con un coágulo de protección. 


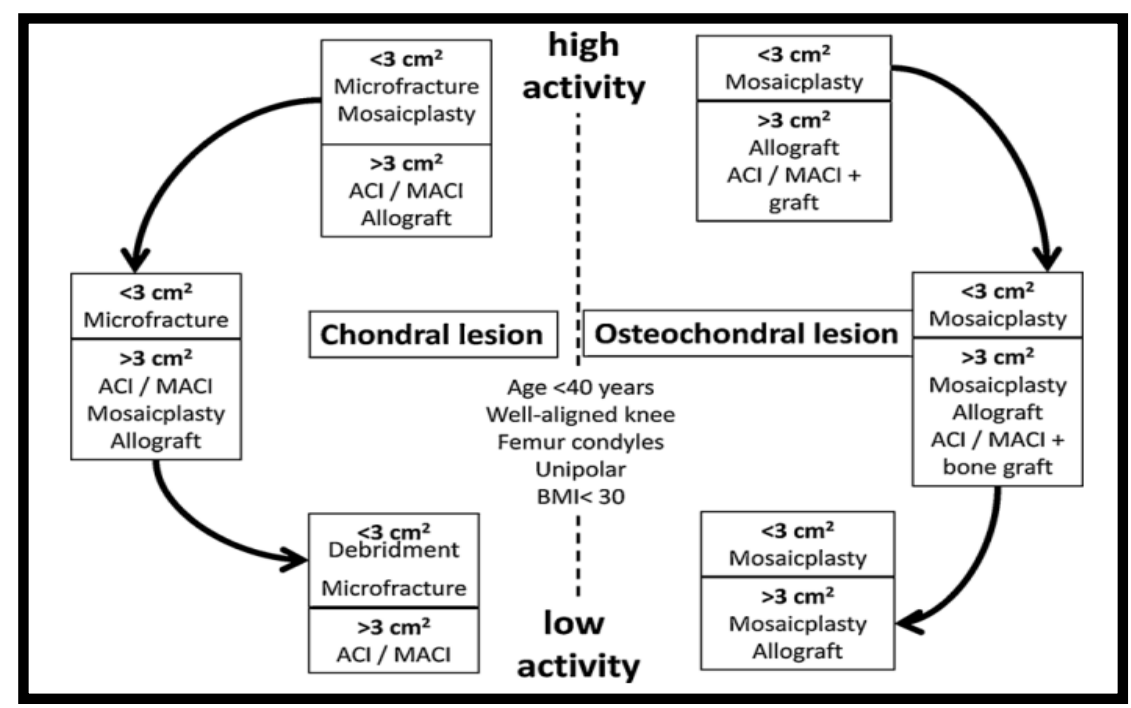

Figura 10. Algoritmo de tratamiento por Vaquero, J., \& Forriol, F. (2012) para lesiones ocndrales de la rodilla. En este algoritmo establecen un límite de $3 \mathrm{~cm}^{2}$.

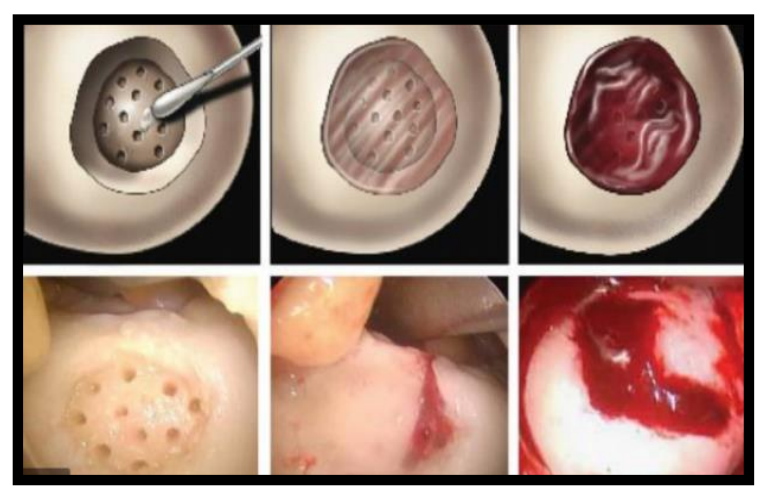

Figura 11: Protección del coágulo con una matriz cartilaginosa para retener el sangrado y la células hematopoyéticas. Imagen obtenida del trabajo de Shive MS., et al. (22)

Tratamiento sustitutivo. Es la actitud que deberíamos tener ante las lesiones más avanzadas que no podamos salvar con tratamiento "auto-reparativos". En estos casos se necesitará ayudar al organismo a rellenar el defecto que se ha creado (generalmente una vez el fargmento es inestable y se ha desprendido). A este respecto, la ciencia no deja de avanzar y mejorar con novedosas técnicas, o aplicando nuevas calidades a las actualmente implantadas.
Injerto óseo autólogo. Se podría tratar de un tratamiento a caballo entre los sustitutivos y los reparadores, ya que consiste en obtener fragmentos pequeños de hueso trabecular e implantarlos en la sueperficie del defecto óseo. Posteriormente, una vez consolidado, podríamos plantearnos la reparación del cartílago mediante otras técnicas sustituivas.

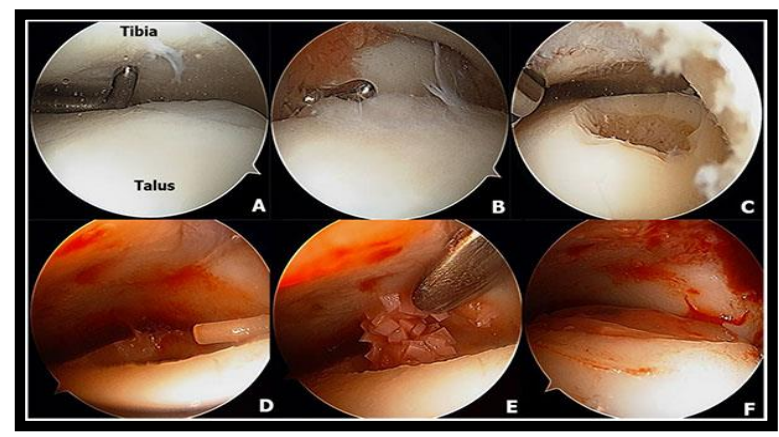

Figura 12: Injerto óseo autólogo para la reparación del defecto astragalino. Imagen obtenida del trabajo de Rungprai C., et al. (23) 
También se ha planteado el uso de aspirado de médula ósea (funcionando de forma similar a la microperforaciones).

Injerto osteocondral. Este tratamiento se reserva para lesiones cartilaginsosas más grandes, sobre todo cuando no podemos salvar los fragmentos, o cuando los anteriores tratamientos han fallado. Las técnicas de extracción/impantación presentan gran dificultad ténica, y puede proceder de diversas fuentes. Una característica del tobillo, es que es una articulación de carga muy congruente, y para acceder al astrágalo a veces será necesaria una osteotomía de peroné. A continuación hablaremos de las virtudes y desventajas de ellas:

Autológo. Se suelen tomar injertos de la parte superior del cóndilo de la rodilla ipsilateral (generalmente del cóndilo externo), independientemente del destino final del injerto). Dentro de esta técnica, existe la mosaicoplastia (24) [Figura 13], que consiste en implantar varios cilindros, con mayor supervivencia global del injerto, sin embargo los espacios que quedan entre ellos jamás se rellenan. Otras características de este tipo de injerto son:

- Baja congruencia articular

- Morbilidad de la zona donante

- Limitado

- Accesible

- Supervivencia alta (corto/medio plazo)
Alogénico. Surgieron después y para resolver la problemática con los injerto autólogos, pero sin embargo, sólo se utilizan cuando es necesario reeemplazar grandes fragmentos osteocondrales y en articulaciones muy congruentes (tobillo). Otras desventajas de esta técnica es que se pueden transmitir enfermedades (injertos conservados "en fresco") y que generalmente tienen poca supervivencia ${ }^{(25)}$.

Sintético. Se trata de una técnica noveda aún y poco estudiada en la que se colocan andamios cilíndricos bifásicos formados por copolímeros sintéticos que facilitan técnica de relleno defecto osteocondral. Sin embargo, presenta problemas como dolor y derrame persistente, así como la no incorporación del cilindro a largo plazo ${ }^{(26)}$.

Cultivo de condrocitos. Se considera un tratamiento prometedor en la cobertura de lesiones condrales aisaldas, pero que se ha incluido en el tratamiento de la osteocondritis en vista de los buenos resultados obtenidos a corto plazo en un metaanálisis para las lesiones osteocondrales de tobillo elaborado por Niemeyer P., et al. ${ }^{(27)}$

- $1^{\text {a }}$ generación o ACl (autologous chondrocyte implantatation). La técnica consiste en biopsiar el cartílago hialino articular en un primer tiempo y tras lavar los condrocitos se cultivan $y$, en un segundo tiempo, se implantan una vez conseguida la cantidad adecuada de células. Finalmente se recubre con una membrana de 
periostio, que se sutura al cartílago sano y se sella con fibrina. Esta técnica dio problemas de hipertrofia del periostio.

- $\underline{2}^{\mathrm{a}}$ y $3^{\mathrm{a}}$ generación o MACl (matrixassociated condrocyte implatation). Para mejorar las complicaciones, se cultivaban en una membrana de colágeno porcino tipo $\mathrm{I} / \mathrm{III}{ }^{(28)}$ o mediante una matriz condroconductora, sin embargo, su aplicación para las osteocondritis requiere que el hueso subcondral esté intacto, por lo que a veces es necesario que se usen en asociación con un injerto de hueso esponjoso.

- $4^{\text {a }}$ generación. Todavía en investigación, se basa sobre todo en la terapia génica y se están buscando otras matrices más duraderas, con mejor integración, mayor resistencia y mejor

Artroplastia. En última instancia se puede plantear el reemplazo articular por una prótesis (e incluso la artrodesis). Esta será la opción de salvamento de los defecto que no hayan podido ser reparados con el resto de técnicas, y cuando la clínica lo indique. En ese contexto, el trabajo de Álvarez E., et al. realiza un algoritmo en función del tamaño de la lesión y opciones de rescate en caso de fallo del tratamiento previo, como último recurso, plantea la opción de la artroplastia ${ }^{(30)}$. No siempre será necesario remmplazar por una prótesis total, se han estudiado resultado

\begin{abstract}
también con prótesis unicompartimentales de rodilla $y$ tobillo con buenos resultados, pero bajo nivel de evidencia. ${ }^{(31)}$.
\end{abstract}

\section{OTROS TRATAMIENTOS}

Por último, hablar de tratamientos englobados quizá dentro de los tratamientos conservadores $\mathrm{O}$ rehabilitadores, con eficacia dudosa y bajo nivel de evidencia. En la literatura podemos encontrar artículos con técnicas prometedoras pero no probados a gran escala, entre los que destacan:

- Infiltraciones intraarticulares con PPRP. Un estudio defiende que es superior a L-PRP (contiene plaquetas $y$ leucocitos) en lesiones osteocondrales del artrágalo, y relacionan esta superioridad con la inhibición de la cascada de señalización osteoblástica PI3K/AKT/AP-1 reduciendo así la secreción de MMP-9 (metaloproteasas) ${ }^{(32)}$. Sin embargo no hay estudios comparativos con otros métodos.

- Ondas de choque. Existe un tratamiento con LIPUS (low-intensity pulse ultrasound) para la osteocondritis del capitellum probado en un estudio, en el que los pacientes mejoraban su periodo de recuperación objetivado por imagen $^{(33)}$. Sin embargo no está claro si es por el efecto directo de las ondas de choque, o por la disminución de la actividad. 


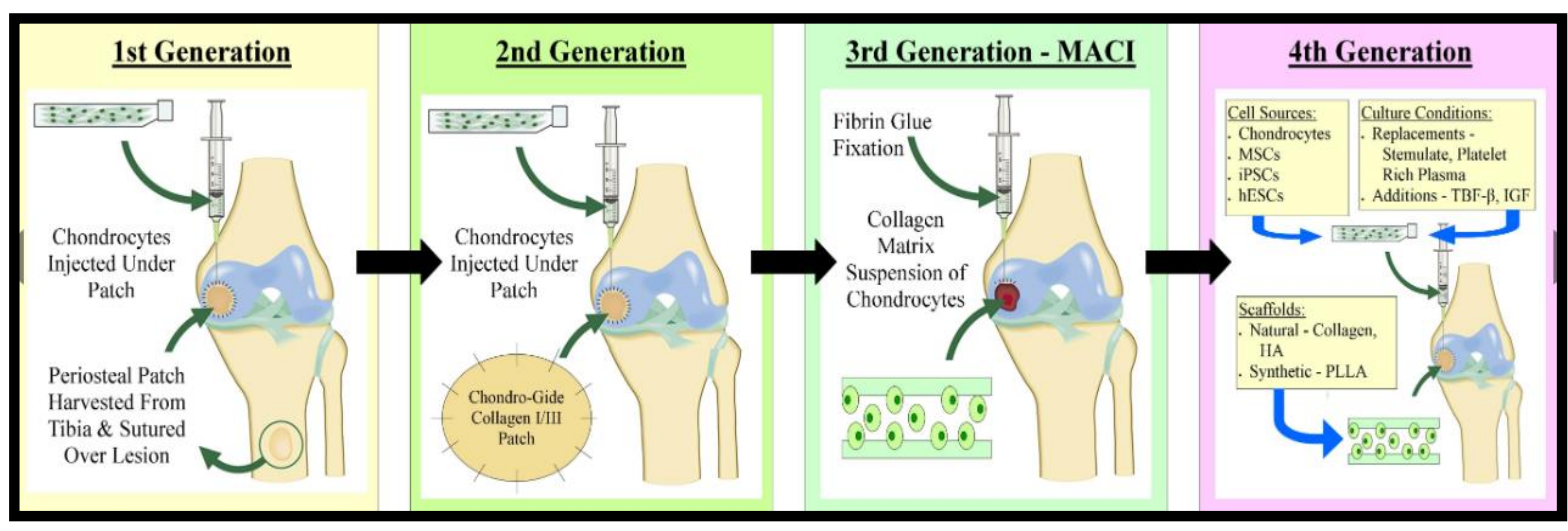

Figura 14. Evolución técnica y en bioingeniería del cultivo de condrocitos para lesiones condrales. Imagen obtenida del trabajo de Davies, R. L., \& Kuiper, N. J. (29)

- Carga temprana. El trabajo Min W., et al. defiende que no encontraron diferencias (escalas EVA y AOFAS) entre los pacientes que cargaron desde el primer día tras tratamiento de lesión osteocondral mediante microfracturas, comparado con aquello que reposaron a partir del mes de la cirugía ${ }^{(34)}$.

\section{DISCUSIÓN}

Para terminar, y como conceptos que tenemos que tener claros con respecto a la osteocondritis, destacamos los siguientes puntos :

- Es una patología que puede pasar desapercibida si no la sospechamos. Cuanto antes se diagnostique, se llevarán a cabo medidas menos agresivas, pues el tratamiento debe ser proporcional a la lesión, para evitar la artrosis.

- En personas jóvenes se prefiere una técnica con buenos resultados a largo plazo.
- En deportistas profesionales optaremos por resultados inmediatos y pronta recuperación.

- No existe actualmente evidencia de que ningún tratamiento sea mejor que otro, aunque en población joven, prácticamente todas las técnicas muestran resultados satisfactorios a corto plazo (incluido el tratamiento conservador) ${ }^{(20,35)}$.

También debemos conocer bien la patología concomitante del paciente, tratarlo en su conjunto, sobre todo para que biomecánicamente el paciente mejore. Primero trataremos posibles deformidades, y posteriormente el defecto condral $u$ osteocondral.

Por todo lo mencionado anteriormente, creemos necesaria la elaboración de ensayos clínicos controlados y con seguimiento a largo plazo para poder establecer guías de práctica clínica con potencia, pues actualmente no disponemos de evidencia de este nivel. Pues suerte para los profesionales de la ciguría ortopédica, a día de hoy podemos encontrar bastantes ensayos en proceso. 


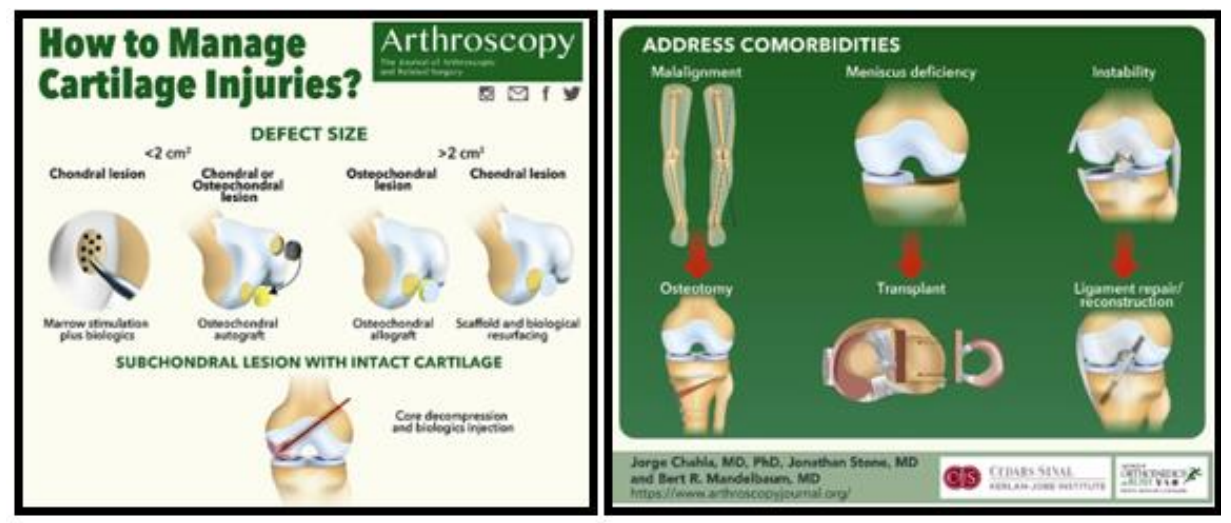

Figura 15. Algortimo de tratamiento propuesto por Chahla J., et al. para lesiones osteocondrales de rodilla, incluido el manejo de comorbilidades. ${ }^{(36)}$

\section{REFERENCIAS BIBLIOGRÁFICAS}

1. Paré A. The case reports and autopsy records of Ambroise Paré. Transl from J P Malgaigne's Oeuvres Complet d'Ambroise Paré Paris 1840 Charles C Thomas; Springf. :113.

2. König F, Brand RA. The classic: On loose bodies in the joint. Clin Orthop Relat Res. 2013;471(4):1107-15.

3. Andriolo L, Crawford DC, Reale D, Zaffagnini S, Candrian C, Cavicchioli A, et al. Osteochondritis Dissecans of the Knee: Etiology and Pathogenetic Mechanisms. A Systematic Review. Cartilage. 2018;

4. Ribbing S. the Hereditary Multjple Epiphyseal Disturbance and Its Consequences for the Aetiogenesis of Locai, Malacjas-Particularly the Osteochondrosis Dissecans. Acta Orthop. 1954;24(1-4):286-99.

5. Shea KG, Jacobs JC, Carey JL, Anderson AF, Oxford JT. Osteochondritis dissecans knee histology studies have variable findings and theories of etiology knee. Clin Orthop Relat Res. 2013;471(4):1127-36.

6. Kessler Jl, Nikizad H, Shea KG, Jacobs JC, Bebchuk JD, Weiss JM. The demographics and epidemiology of osteochondritis dissecans of the knee in children and adolescents. Am J Sports Med. 2014;42(2):320-6.

7. Kessler Jl, Weiss JM, Nikizad $\mathrm{H}$, Gyurdzhyan S, Jacobs JC, Bebchuk JD, et al. Osteochondritis dissecans of the ankle in children and adolescents: Demographics and epidemiology. Am J Sports Med. 2014;42(9):2165-71.

8. Kessler JI, Jacobs JC, Cannamela PC, Weiss JM, Shea KG. Demographics and Epidemiology of Osteochondritis Dissecans of the Elbow Among Children and Adolescents. Orthop J Sport Med. 2018;6(12):1-6.

9. Grimm NL, Weiss JM, Kessler JI, Aoki 
SK. Osteochondritis dissecans of the knee: Pathoanatomy, epidemiology, and diagnosis. Clin Sports Med [Internet]. 2014;33(2):181-8. Available from: http://dx.doi.org/10.1016/j.csm.2013 .11 .006

10. García Cañas $R$, Sánchez Carrillo $M$, Rodríguez Moro C, AJ GL, I PP, Areta Jiménez F. Rarefacción y fragmentación del capitellum pediátrico. Caso clínico de la Enfermedad de Panner en el Hospital Central de la Defensa. Sanid Mil. 2019;75(2):113-5.

11. van den Ende KIM, Keijsers R, van den Bekerom MPJ, Eygendaal D. Imaging and classification of osteochondritis dissecans of the capitellum: X-ray, magnetic resonance imaging or computed tomography? Shoulder Elb. 2019;11(2):129-36.

12. Bauer KL, Polousky JD. Management of Osteochondritis Dissecans Lesions of the Knee, Elbow and Ankle. Clin Sports Med [Internet]. 2017;36(3):469-87. Available from: http://dx.doi.org/10.1016/j.csm.2017 .02 .005

13. BERNDT AL, HARTY M. Transchondral fractures (osteochondritis dissecans) of the talus. J Bone Joint Surg Am. 1959;41-A:988-1020.

14. Brittberg $M$, Winalski CS. Evaluation of cartilage injuries and repair. In: Journal of Bone and Joint Surgery Series A. Journal of Bone and Joint Surgery Inc.; 2003. p. 58-69.
15. Nepple JJ, Milewski MD, Shea KG. Research in Osteochondritis Dissecans of the Knee: 2016 Update. J Knee Surg. 2016;29(7):533-8.

16. Masquijo J, Kothari A. Juvenile osteochondritis dissecans (JOCD) of the knee: Current concepts review. EFORT Open Rev. 2019;4(5):201-12.

17. Salci $L$, Ayeni $O$, Abouassaly $M$, Farrokhyar F, D'Souza JA, Bhandari M, et al. Indications for surgical management of osteochondritis dissecans of the knee in the pediatric population: a systematic review. J Knee Surg. 2014;27(2):147-55.

18. Andriolo $\mathrm{L}$, Candrian $\mathrm{C}$, Papio $\mathrm{T}$, Cavicchioli A, Perdisa F, Filardo G. Osteochondritis Dissecans of the Knee - Conservative Treatment Strategies: A Systematic Review. Cartilage. 2019;10(3):267-77.

19. Vaquero J, Forriol F. Knee chondral injuries: Clinical treatment strategies and experimental models. Injury [Internet]. 2012;43(6):694-705. Available from: http://dx.doi.org/10.1016/j.injury.20 11.06 .033

20. Abouassaly $M$, Peterson D, Salci $L$, Farrokhyar F, D'Souza J, Bhandari M, et al. Surgical management of osteochondritis dissecans of the knee in the paediatric population: A systematic review addressing surgical techniques. Knee Surgery, Sport Traumatol Arthrosc. 2014;22(6):1216-24.

21. Bojanić I, Smoljanović T, Dokuzović S. 
Osteochondritis dissecans of the elbow: Excellent results in teenage athletes treated by arthroscopic debridement and microfracture. Croat Med J. 2012 Feb;53(1):40-7.

22. Shive MS, Stanish WD, McCormack R, Forriol F, Mohtadi N, Pelet $S$, et al. BST-CarGel ${ }^{\circledR}$ Treatment Maintains Cartilage Repair Superiority over Microfracture at 5 Years in a Multicenter Randomized Controlled Trial. Cartilage. 2015 Apr 27;6(2):6272.

23. Rungprai C, Tennant JN, Gentry RD, Phisitkul P. Management of Osteochondral Lesions of the Talar Dome. Open Orthop J. 2017;11(1):743-61.

24. Hangody L, Füles P. Autologous osteochondral mosaicplasty for the treatment of full-thickness defects of weight-bearing joints: Ten years of experimental and clinical experience. In: Journal of Bone and Joint Surgery - Series A. Journal of Bone and Joint Surgery Inc.; 2003. p. 25-32.

25. Murawski CD, Kennedy JG. Operative treatment of osteochondral lesions of the talus. J Bone Jt Surg - Ser A [Internet]. 2013;95(11):1045-54. Available from: http://dx.doi.org/10.1016/S00219355(13)72276-8

26. Carmont MR, Carey-Smith R, Saithna A, Dhillon M, Thompson P, Spalding T. Delayed Incorporation of a TruFit Plug: Perseverance Is Recommended. Arthrosc - J Arthrosc Relat Surg
[Internet]. 2009 Jul [cited 2020 Mar 3];25(7):810-4. Available from: http://www.ncbi.nlm.nih.gov/pubme d/19560648

27. Niemeyer P, Salzmann G, Schmal H, Mayr $H$, Südkamp NP. Autologous chondrocyte implantation for the treatment of chondral and osteochondral defects of the talus: A meta-analysis of available evidence. Knee Surgery, Sport Traumatol Arthrosc [Internet]. 2012 Aug [cited 2020 Mar 3];20(9):1696-703. Available from: http://www.ncbi.nlm.nih.gov/pubme d/22037894

28. Zanon G, Vico G Di, Marullo M. Osteochondritis dissecans of the talus. Joints. 2017 Sep 19;02(03):11523.

29. Davies RL, Kuiper NJ. Regenerative Medicine: A Review of the Evolution of Autologous Chondrocyte Implantation (ACl) Therapy. Bioengineering. 2019;6(1):1-16.

30. Álvarez E, Ripoll PL, Restrepo $A$, Forriol F. Revisión de la reparación del cartílago. Posibilidades y resultados. Trauma (Spain). 2010;21(2):117-34.

31. van Bergen CJA, Reilingh $M L$, van Dijk $\mathrm{CN}$. Tertiary osteochondral defect of the talus treated by a novel contoured metal implant. Knee Surgery, Sport Traumatol Arthrosc. 2011 Jun;19(6):999-1003.

32. Wei F, Wang Z. Effectiveness and mechanism of pure platelet-rich 
plasma on osteochondral injury of talus. Zhongguo Xiu Fu Chong Jian Wai Ke Za Zhi. 2019 May 15;33(5):555-62.

33. Kusano $H$, Furushima $K$, Mitsui $Y$, Miyamoto A, Urata D, Serita $Y$, et al. 7. Usefulness of Low-Intensity Pulsed Ultrasound (LIPUS) for the Treatment of Osteochondritis Dissecans (OCD) of the Humeral Capitellum. J Orthop Trauma [Internet]. 2016 Aug [cited 2020 Mar 4];30(8):S3-4. Available from:

http://www.ncbi.nlm.nih.gov/pubme d/27441768

34. Wei M, Wei Y, Liu Y. Effects of Early Weightbearing on Microfracture Treatment of Osteochondral Lesions of Talus with Subchondral Bone Defects. Curr Med Sci [Internet]. 2019 Feb 1 [cited 2020 Mar 4];39(1):88-93.
Available from: http://www.ncbi.nlm.nih.gov/pubme d/30868496

35. Ananthaharan A, Randsborg $\mathrm{PH}$. Epidemiology and patient-reported outcome after juvenile osteochondritis dissecans in the knee. Knee [Internet]. 2018;25(4):595-601. Available from: https://doi.org/10.1016/j.knee.2018. 02.005

36. Chahla J, Stone J, Mandelbaum BR. How to Manage Cartilage Injuries? Arthrosc - J Arthrosc Relat Surg [Internet]. 2019 Oct 1 [cited 2020 Mar 4];35(10):2771-3. Available from: http://www.ncbi.nlm.nih.gov/pubme d/31604490 